\title{
Journal of Real-Time Image Processing: fourth issue of volume 6
}

\author{
Nasser Kehtarnavaz $\cdot$ Matthias F. Carlsohn
}

Published online: 26 October 2011

(C) Springer-Verlag 2011

As was mentioned in an earlier editorial, JRTIP is now included in Thomson Reuters Science Citation Index. The 2010 impact factor appearing on the journal website stands at 0.962 and is ranked 72 out of 108 in the Artificial Intelligence category, 124 out of 247 in the Engineering, Electrical and Electronic category and 8 out of 19 in the Imaging Science and Photographic Technology category.

The editorial board of JRTIP will be meeting in Brussels in April of next year while attending the SPIE Conference on Real-Time Image and Video Processing to discuss further steps to be taken to improve this index. It is observed that the academic community is now paying more attention to the real-time aspects of image and video processing. We expect this index to rise next year due to this recognition of JRTIP and the quality of papers published.

This regular issue includes 7 papers covering algorithmic, software, and hardware aspects of real-time image and video processing to enable real-time throughput with acceptable or tolerable loss in performance.

The first paper by Huang et al. presents the real-time software implementation of a multimedia, tele-medicine system by considering video streaming from both video servers in hospitals and webcams on patients. It also considers transmission of vital bio-signs including heart rates and blood pressure. All these data are transmitted over a

\footnotetext{
N. Kehtarnavaz $(\bowtie)$

Department of Electrical Engineering,

University of Texas at Dallas, Dallas, USA

e-mail: kehtar@utdallas.edu

M. F. Carlsohn

Computer Vision and Image Communication Engineering

and Consultancy Dr. Carlsohn, Bremen, Germany

e-mail: matthias.carlsohn@t-online.de
}

3G-wireless communication link to hand-held devices used by physicians and nurses.

The second paper by Ranjit et al. addresses a blockmatching algorithm based on a hexagon-diamond search procedure in order to estimate motion vector parameters. This search is shown to involve fewer search points thus reducing the computational complexity while producing similar performances in terms of image quality as compared to other algorithms.

The third paper by Hamshari et al. discusses a real-time framework for the detection and tracking of the face and eyes of drivers during several driving scenarios, thus allowing for an understanding of their visual search pattern. A confidence parameter is introduced to help minimize the errors produced by incorrect matches and allow more effective tracking.

The fourth paper by Petrosino et al. introduces a realtime streaming system for multimedia based on real-time operating systems. Tests are reported to measure jittering, one of the most important aspects of such systems. The proposed system is shown to achieve good performance both for simulated and real-world cases.

The fifth paper by Georgoulas et al. covers a hardware engine for the computation of stereo disparity map in realtime. This engine is based on a single FPGA device with an operating frequency of $138 \mathrm{MHz}$. The hardware is shown to provide accurate disparity map computation at a rate of nearly 440 frames per second, given a stereo image pair with a disparity range of 80 pixels and $640 \times 480$ pixels spatial resolution.

The sixth paper by Gembris et al. is on the real-time data processing aspect of neurofeedback experiments in functional magnetic resonance imaging. More specifically, it addresses the computational bottleneck associated with the use of connectivity analysis. The paper shows the performance benefits that are gained. 
The seventh and final paper by Chen et al. presents the design of real-time implementable full-reference image/ video quality algorithms based on the Structural SIMilarity (SSIM) and multi-scale SSIM (MS-SSIM) indices. The experimental results reported indicate that the performance of these algorithms is in par with that of SSIM and MS-SSIM while involving much lower computational complexity.
As always, we thank all of the Associate Editors and reviewers for their ongoing efforts and contributions to JRTIP. At the end, we wish to express our thanks to the Associate Editors Eiji Nakamura and Luciano da Fontoura Costa who are stepping down from the Editorial Board at the end of this year. We thank them for their contributions to JRTIP and wish them all the best. 